Document downloaded from:

http://hdl.handle.net/10251/49277

This paper must be cited as:

Conde Castellanos, PE.; González Martínez, AJ.; Hernández Hernández, L.; Bellido, P.; Iborra Carreres, A.; Crespo Navarro, E.; Moliner Martínez, L.... (2014). Results of a combined monolithic crystal and an array of ASICs controlled SiPMs. Nuclear Instruments and Methods in Physics Research Section A: Accelerators, Spectrometers, Detectors and Associated Equipment. 734:132-136. doi:10.1016/j.nima.2013.08.079.

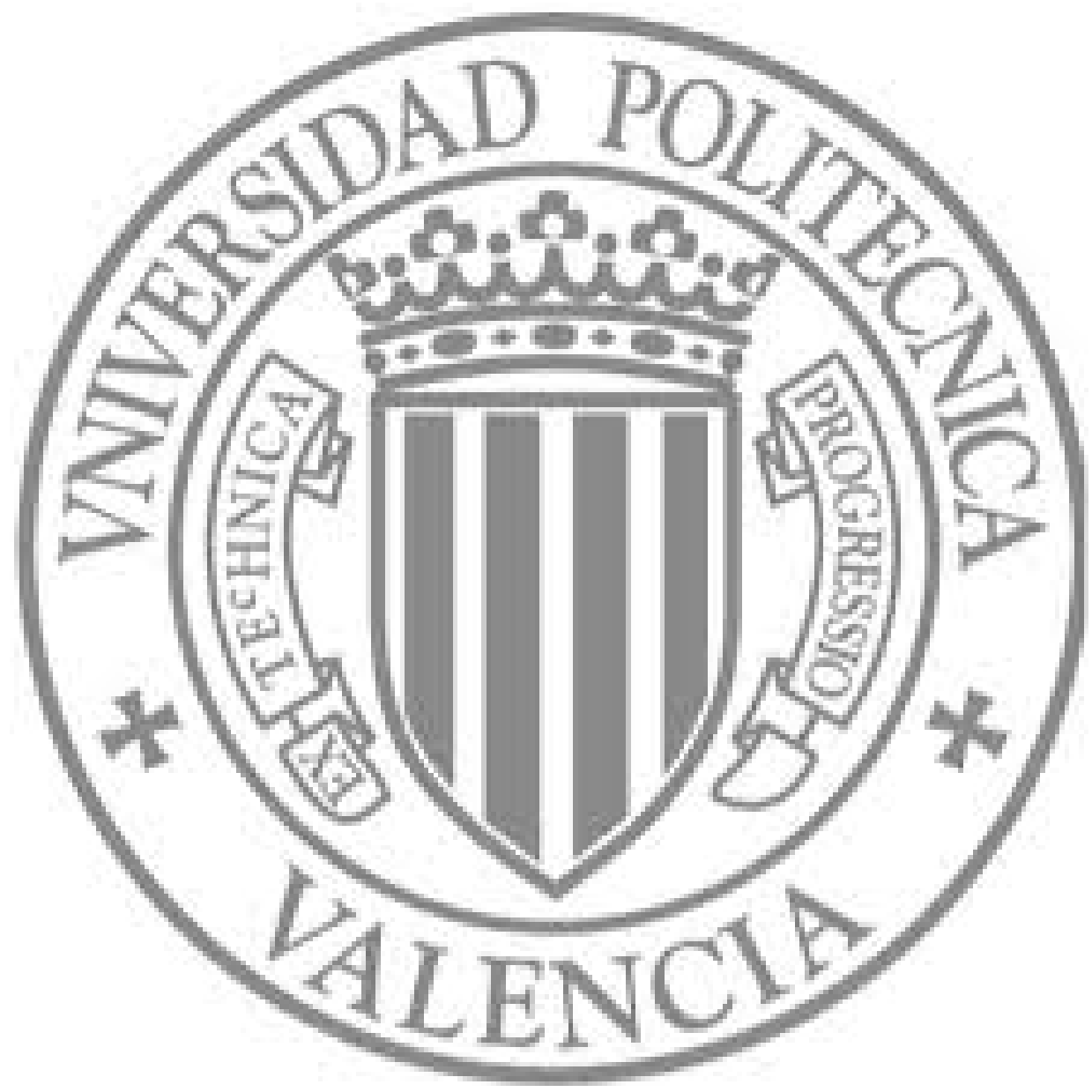

The final publication is available at

http://dx.doi.org/10.1016/j.nima.2013.08.079

Copyright Elsevier 


\title{
Results of a combined monolithic crystal and an array of ASICs controlled SiPMs
}

\author{
P. Conde ${ }^{\mathrm{a}}$, A.J. González ${ }^{\mathrm{a}, *}$, L. Hernández ${ }^{\mathrm{a}}$, P. Bellido ${ }^{\mathrm{a}}$, A. Iborra ${ }^{\mathrm{a}}$, E. Crespo $^{\mathrm{a}}$, L. Moliner ${ }^{\mathrm{a}}$, M.J. Rodríguez-Álvarez ${ }^{\mathrm{a}}$, F. Sánchez $^{\mathrm{a}}$, \\ M. Seimetz ${ }^{\mathrm{a}}$, A. Soriano ${ }^{\mathrm{a}}$, L.F. Vidal ${ }^{\mathrm{a}}$, J.M. Benlloch ${ }^{\mathrm{a}}$ \\ ${ }^{a}$ Institute for Instrumentation in Molecular Imaging (I3M). Centro Mixto UPV - CSIC - CIEMAT. Camino de Vera s/n, 46022, Valencia, Spain.
}

\begin{abstract}
In this work we present the energy and spatial resolutions we have obtained for a $\gamma$-ray detector based on a monolithic LYSO crystal coupled to an array of $256 \mathrm{SiPMs}$. Two crystal configurations of the same trapezoidal shape have been tried. In one approach all surfaces were black painted but the exit one facing the photosensor array which was polished. The other approach included a retroreflector (RR) layer coupled to the entrance face of the crystal powering the amount of transmitted light to the photosensors. Two coupling media between the scintillator and the SiPM array were used, namely direct coupling by means of optical grease and coupling through an array of light guides. Since the same operational voltage was supplied to the entire array, it was needed to equalize their gains before feeding their signals to the Data Acquisition system. Such a job was performed by means of 4 scalable Application Specific Circuits (ASICs). An energy resolution of about $24.4 \%$ has been achived for the direct coupling with the RR layer together with a spatial resolution of approximately $2.9 \mathrm{~mm}$ at the detector center. With the light guides coupling the effects of image compression at the edges are significantly minimized, but worsening the energy resolution to about $33.1 \%$ with a spatial resolution nearing $4 \mathrm{~mm}$ at the detector center.
\end{abstract}

Keywords:

Monolithic crystals, SiPMs, ASIC,

\section{Introduction}

Photosensors based on Silicon Photomultipliers (SiPMs) are ${ }_{28}$ considered good candidates to substitute the well established ${ }_{29}$ Photomultiplier Tube (PMT) technology ${ }^{(1,2)}$. SiPMs are very fast, have high gain, almost unaffected by magnetic fields ${ }^{(3)}{ }_{31}^{30}$ being easy to manufacture when compared to PMTs. We intend to use arrays of SiPMs for the design of Positron Emission Tomography (PET) detectors compatible with Magnetic Resonance (MR) systems. It has been pointed out the convenience of simultaneously obtain PET and MR images ${ }^{(4)}$.

Conventional whole-body PET systems, even those enabling the Time of Flight (TOF) determination, can hardly reach a spatial resolution better than $5 \mathrm{~mm}^{(5)}$. SiPMs also enable determination of TOF of the $511 \mathrm{keV}$ annihilation photons ${ }^{(6)}$. Since whole-body systems can suffer from patient motion effects or restricted access to the imaging organ, SiPMs based detector together with monolithic scintillators are best suitable for dedicated systems as are animal or brain detectors where the physical limits of PET scanners are reached. SiPMs exhibit their best performance in reduced active areas where the intrinsic dark counts are minimized. This type of detectors, in contrast to PMTs, account for moderate noise effects due to thermal excitation which is amplified and output as dark counts.

We propose a detector block containing two main components namely a SiPM array and a single monolithic crystal.

\footnotetext{
* Institute for Instrumentation in Molecular Imaging (I3M)

Email address: agonzalez@i3m.upv.es (A.J. González)
} coordinates, thus including the Depth of Interaction (DOI) ${ }^{(7)}$ The knowledge of the DOI can be obtained through the second centered moment, namely the light spread (see Fig. 1). Another important feature when dealing with continuous crystals is that their final spatial resolution is not limited by the pixel size as it is the case of crystal arrays, but rather by the accuracy in the determination of the center of gravity of the light distribution ${ }^{(8)}$.

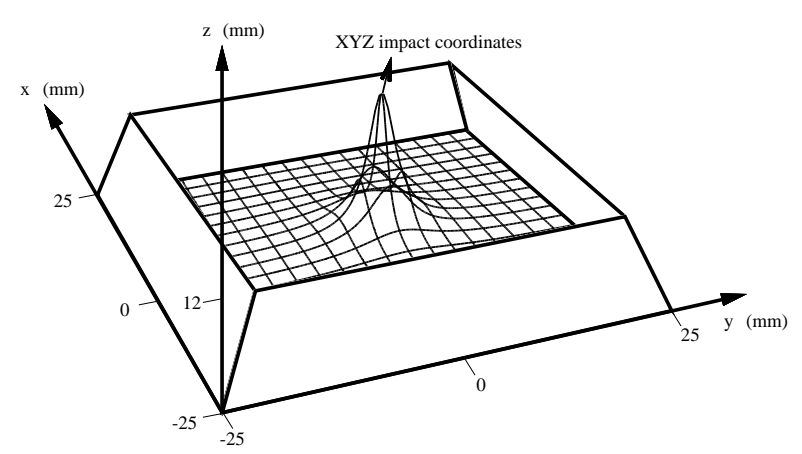

Figure 1: Light distribution produced inside a monolothic LYSO crystal. Analysis of statistical moments can provide information about the three-dimensional impact coordinates.

In this work we present a study of the spatial resolution and border effect of the suggested detector block. We have also analyzed the energy resolution of the different configurations. 
In particular, we have used two coupling methods between the 92 monolithic crystal and the SiPMs array namely direct coupling 93 by means of optical grease and coupling via light guides ${ }^{(9)}$. In 94 addition to the coupling method, we also evaluated the use of 95 a retroreflector (RR) layer located on the entrance face of the 96 crystal in order to increase the amount of light transferred to 97 the photosensor array without disturbing the light distribution. 98

\section{Materials and Methods}

\subsection{Monolithic crystal and optical devices}

Since the dark noise in SiPM devices is proportional to its 103 active area, SiPMs with $1 \times 1 \mathrm{~mm}^{2}$ active area were selected ${ }_{104}$ (Hamamatsu Photonics, model S10362-11, Japan). An array ${ }_{105}$ of $256 \mathrm{SiPM}$, with $3 \mathrm{~mm}$ pitch, has been developed to cover the whole scintillator exit surface ${ }^{(10)}$. These detectors were mounted on a Printed Circuit Board (PCB) with a Z-planarity average and maximum deviation along the 256 SiPMs of $33 \mu \mathrm{m}$ and $87 \mu \mathrm{m}$, respectively. Their $X$ and $Y$ position accuracy was of about $50 \mu \mathrm{m}$.

Two trapezoidal crystals manufactured by Proteus (Ohio, USA) of the same dimensions $(50 \times 50$ for the exit face, $40 \times 40$ for the entrance face and $12 \mathrm{~mm}$ thick) have been tried. In the first approach, all the crystal faces were black painted but the exit one to the photosensor array which was polished. The other approach included the RR layer coupled to the entrance face of the crystal which was also polished. Thus, the formerly absorbed light was retroreflected powering the amount of transmitted light to the photosensors.

The two types of coupling experiments counted with the direct coupling between the scintillator and the SiPM array using optical grease (Rhodorsil Paste 7) and the use of optical guides. These guides are optical concentrators which funnel the light from a square entrance area of $3 \times 3 \mathrm{~mm}^{2}$ into a smaller output area, also squared but of $1 \times 1 \mathrm{~mm}^{2}$ matching the SiPM active area. They work as total internal reflection (TIR) devices ${ }^{(11)}{ }^{106}$ These devices constrain the acceptance angle of the incoming light to approximately $16^{\circ}$, compared to $54.6^{\circ}$ when using the direct coupling.

\subsection{ASIC readout}

The SiPMs matrix is readout through 4 identical Application Specific Integrated Circuit (ASIC) chips called AMIC ${ }^{(12)}$, (see sketch in Fig. 2). Each AMIC reads 64 SiPM inputs and outputs up to 8 signals each. The AMIC chip is fully scalable: $4_{111}$ AMICS are coupled together to read 256 SiPMs in parallel but ${ }_{112}$ working as a single unit. Each AMIC first makes up to 8 copies $_{113}$ of the input signals from each SiPM, which are then multiplied ${ }_{114}$ by a different weight depending on the copy and SiPM posi- ${ }_{115}$ tion. Finally, all the input signals are added forming 8 linear $_{116}$ combinations of the 256 input signals. The weights are programmable via an $\mathrm{I} 2 \mathrm{C}$ bus and stored in 8 -bit registers. Selecting the proper set of weights allows one to estimate many characteristic parameters of the light distribution, e.g. the centroids of the light distribution, their standard deviations, skew-117 ness, etc ${ }^{(13)}$, but also compensate gain differences between in-118 put signals.
Although each SiPM has an optimized bias voltage, provided by the manufacturer, the whole SiPM array was powered at the same voltage level, which produces different gains on each SiPM output signal. To compensate these differences, the ASICs programmable coefficients were equalized using the manufacturer information and tested with ${ }^{22} \mathrm{Na}$ uniform radiation over the scintillation crystal ${ }^{(14,15)}$. This is crucial in order to compute an accurate energy resolution of the system and therefore achieve a good spatial detector resolution. Figure 3Top shows the uniform acquisition when a black scintillator is directly coupled to the SiPM array without the gain compensation. Figure 3-Bottom depicts the same type of acquisition but when equalizing the different gains through the ASIC coefficients.

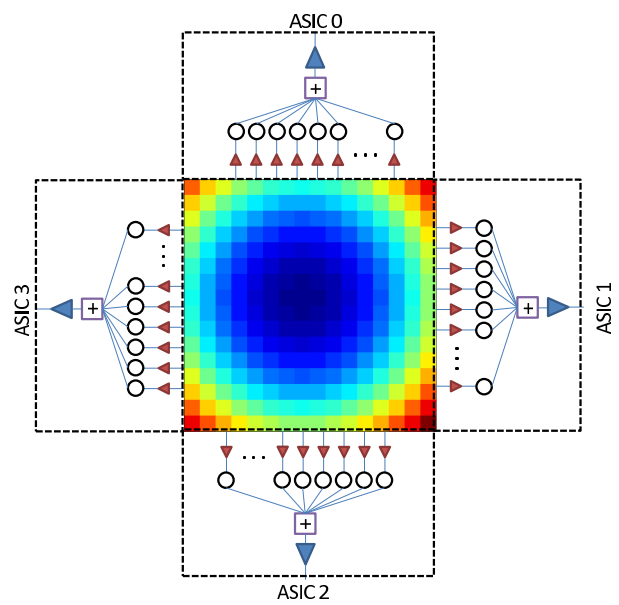

Figure 2: Sketch of 4-ASIC readout. Outputs from each ASIC are added, so that only one signal is digitalized for each moment

\subsection{Center of gravity based spatial resolution}

The scintillation light distribution produced in monolithic LYSO crystals is ideally described by the inverse square law. The projection of the normalized one dimensional inverse square law onto the abscissa $X$, can be written as:

$$
J\left(x, x_{c}\right)=\frac{J_{c}}{\pi} \frac{z_{c}-z_{0}}{\left(\left(x-x_{c}\right)^{2}+\left(z_{c}-z_{0}\right)^{2}\right)},
$$

where $x_{c}, z_{c}$ are the impact coordinates, $J_{c}$ the number of scintillation light photons generated at the impact point and $z_{0}$ the plane of photodetector entrance window. This light distribution model (LDM) is subsequently discretized ${ }^{(16)}$ at the SiPMs array (as it is shown in Fig.4), which is mathematically described for a given SiPM by the integral:

$$
\alpha_{n}\left(x_{c}\right)=\int_{n \cdot d-t / 2}^{n \cdot d+t / 2} J\left(x, x_{c}\right) d x,
$$

where $n$ is the considered SiPM number, $d$ is the SiPMs pitch and $t$ is the size of one SiPM. The set of numbers obtained after integration, are: 


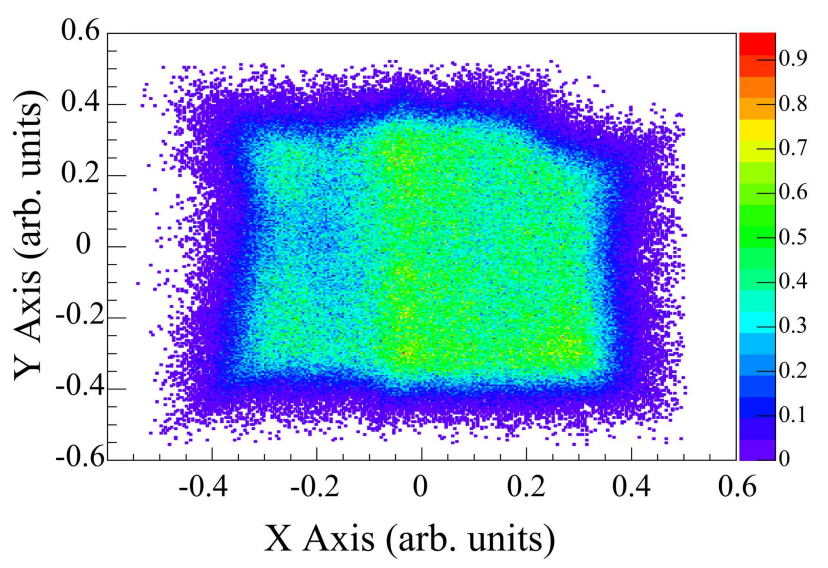

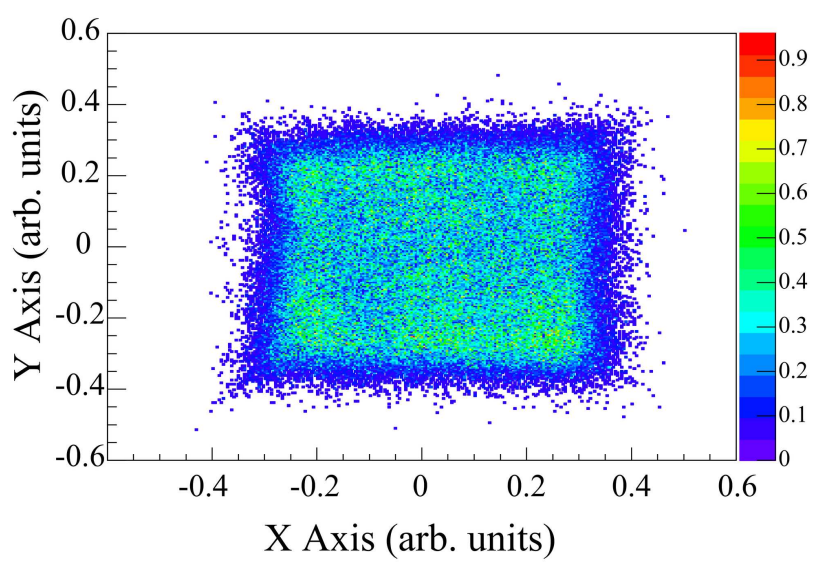

Figure 3: Acquisition of uniform ${ }^{22} \mathrm{Na}$ radiation with uncalibrated coefficients (Top) and equalized coefficients (Bottom). The XY representation reduces when equalizing as an effect of the programmed coefficients range.

$\alpha_{n}\left(x_{c}\right)=\frac{J_{c}}{\pi}\left(\operatorname{Arctan}\left(\frac{n \cdot d+\frac{t}{2}-x_{c}}{z_{c}-z_{0}}\right)-\operatorname{Arctan}\left(\frac{n \cdot d-\frac{t}{2}-x_{c}}{z_{c}-z_{0}}\right)\right)_{139}^{138}$

The Center of Gravity (CoG) of recorded impacts, is then ${ }^{141}$ computed with the equation:

$$
x_{C o G}\left(x_{c}\right)=\frac{\sum_{n=-N}^{N} \alpha_{n}\left(x_{c}\right) \cdot n}{\sum_{n=-N}^{N} \alpha_{n}\left(x_{c}\right)}
$$

where $2 N$ is the total number of SiPM in every row of the $X^{150}$ direction. Since $t<d$, the measured CoG differs from the real impact point everywhere except for $x_{c}=n \cdot d$ and $x_{c}=n \cdot d / 2^{151}$ for $N \rightarrow \infty$. Ergo, a periodic error due to the dead area on the ${ }_{152}$ computed CoG with period $d$ is generated. The magnitude of ${ }_{153}$ the error is also dependent on the width of the LDM, thus with ${ }_{154}$ DOI.

For a finite set of detectors, in our detector block only $16_{156}$ SiPMs in $\mathrm{X}$ direction for a given row contribute to the $\mathrm{LDM}_{157}$

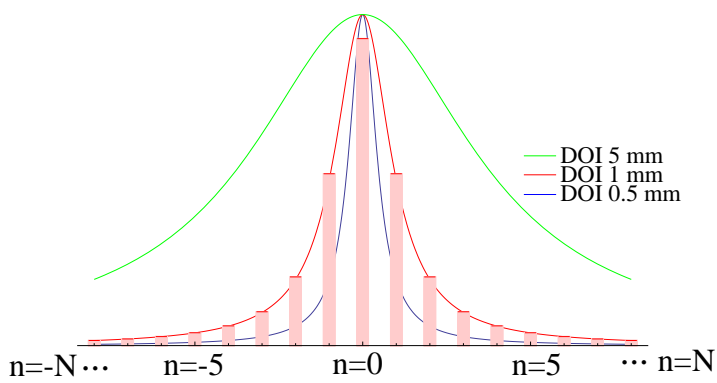

Figure 4: Normalized LDMs for different DOI. Red bars are the digitalized values for $\mathrm{DOI}=1 \mathrm{~mm}$.

digitalization, the measured $\mathrm{CoG}$ differs from the real CoG except for $x_{c}=0$ since the distribution symmetry is broken. It is seen that the truncation of the signal distribution at the detector limits compresses the identity $y=x^{(17)}$, as it can be seen in Fig.5 (a).

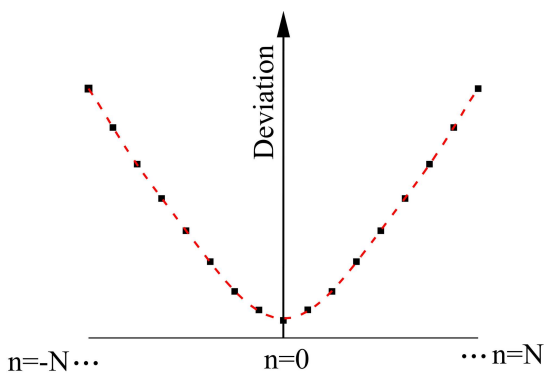

Figure 5: Deviation of CoG measured values along the $X$ axis. An average ${ }^{(18)}$ value of $4.3 \mathrm{~mm}$ for the DOI has been used for the simulation.

\section{Experimental results}

The experimental acquisitions were carried out using two detector modules working in coincidence. The SiPMs array in the detector block under analysis was always gain-equalized as described above. The reference detector was a detector block using an H8500 (Hamamatsu Photonics, Japan) position sensitive PMT and a monolithic crystal with a thickness of $12 \mathrm{~mm}$. ${ }^{22} \mathrm{Na}$ source of $1 \mathrm{~mm}^{2}$ of diameter was utilized. The source was collimated using a Tungsten block of $3 \mathrm{~cm}$ thickness and an aperture of $1.2 \mathrm{~mm}$ in diameter. The source and collimator were moved across the studied detector block in steps of $5 \mathrm{~mm}$ with a digital controlled motorized positioning system (OWIS, Germany) and, thus, up to 9 data points were analyzed along the $X$ direction.

\subsection{Energy resolution}

The energy resolution was determined for the four experimental set-ups namely, direct coupling and coupling through light guides with and without RR. Such a resolution was evaluated using the collimated ${ }^{22} \mathrm{Na}$ source placed at the detector center. A squared region of interest surrounding the imaged source of approximately $5 \times 5 \mathrm{~mm}^{2}$ was considered during the 


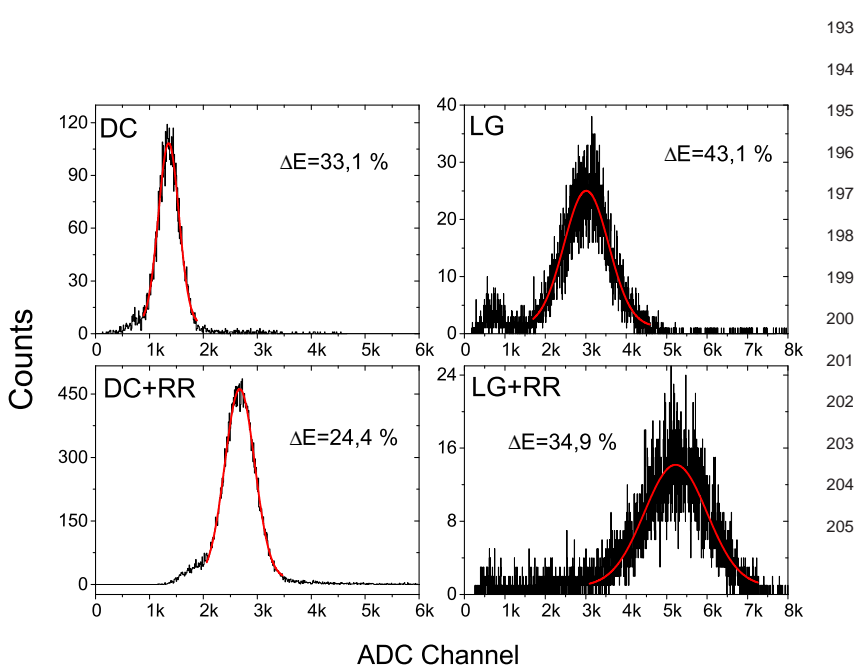

data analysis. The bias voltage of the SiPM array was set to 190

Figure 6: Energy spectra and Gaussian fit to the $511 \mathrm{keV}$ peak. DC stands for the direct coupling and LG for the light guides coupling, respectively.

Figure 6 shows the results of energy resolution for the four configurations and a Gaussian fit to the experimental data depicted with a solid line. When the RR is introduced in the system, we observe an increase of the energy gain, which is of about a factor 2 for both cases, direct coupling and coupling using the light guides.

Direct coupling constrains the acceptance angle (AA) of the incoming light to the photosensors to $54.6^{\circ}$, while light guides to approximately $16^{\circ}$. The solid angle that covers the scintillation light is related to the acceptance angle through the equation:

$$
\Omega=\pi \cdot \sin ^{2}(A A) .
$$

In terms of the fraction of scintillation light that reaches the $\mathrm{e}^{207}$ entrance window of the SiPM array, a $16.6 \%$ is expected in ${ }^{208}$ the case of direct coupling and $1.9 \%$ for coupling with opti- ${ }^{209}$ cal guides. Transmission efficiency of light guides is of about ${ }^{210}$ $70 \%{ }^{(10)}$. In the case of direct coupling, the fraction of sampled scintillation light is even smaller, since $89.9 \%$ of the pho- ${ }_{211}$ todetection surface is dead area, so approximately $1.8 \%$ is the amount of light digitalized with respect to the total scintillation ${ }_{212}$ light produced in the crystal. Such effect degrades the signal $_{213}$ to noise ratio, affecting energy resolution and also the spatial ${ }_{214}$ resolution as it will be observed later.

In these plots it is also observed that the light guides, al-216 though they allow to focus the scintillator light from a $3 \times 3 \mathrm{~mm}^{2}{ }_{217}$ region to the SiPM active area of $1 \times 1 \mathrm{~mm}^{2}$, they result on a 218 poorer energy resolution compared to the case of direct cou-219 pling either with or without the RR. This effect is most likelyz20 due to the transmission losses which worsens the light collec-221 tion.

\subsection{Spatial resolution}

Figure 7 shows the variation of spatial resolution along the $X$ axis, for the 9 measured point positions and for the four experimental configurations. In all cases, the variation is related with the predicted model depicted in Fig. 5, and the spatial resolution values are correlated with the amount of light that reaches the photosensors entrance window. The best spatial resolutions can be found at the center of the detector, inasmuch as is the unique point where the symmetry of the light distribution is preserved. For the direct coupling a spatial resolution of 4.9 $\pm 0.1 \mathrm{~mm}$ without RR and $2.9 \pm 0.1$ with RR is obtained. In the experiment with coupling through light guides, a spatial resolution at the center of $6.4 \pm 0.1$ without $R R$ and $4.4 \pm 0.1$ with $\mathrm{RR}$ was found. The restricted acceptance angle for light guides coupling worses the spatial resolution, but improves with the addition of the RR layer.

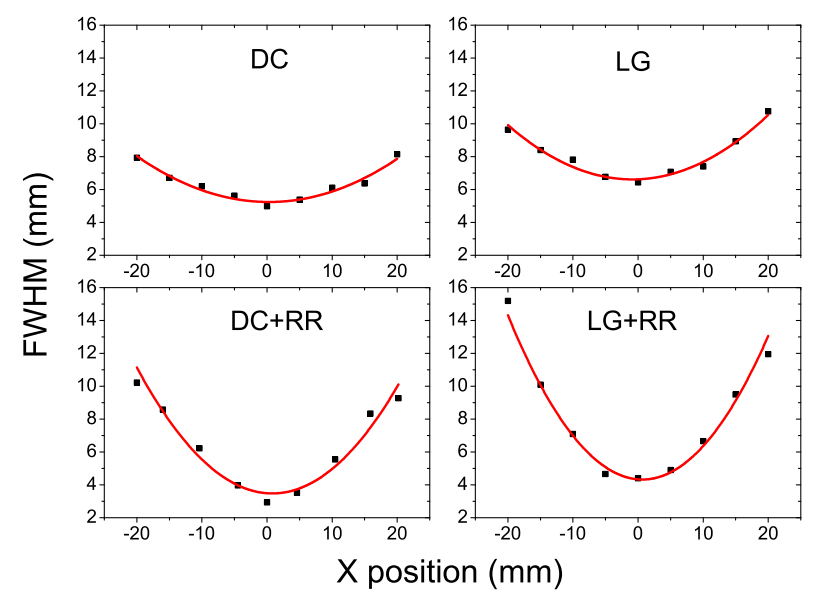

Figure 7: Variation over the $X$ axis of the spatial resolution for the four experimental set-ups.

Although the energy and spatial resolution worsens with the use of the light guides, the effects of compression at the edges are significantly minimized, as shown in Fig.8. However, the increase of scintillation light with the RR pronounces the border effects.

\section{Conclusions}

In this work we have described different $\gamma$-ray detector block configurations for their use in PET systems, compatible with MR scanners.

An array of $256 \mathrm{SiPMs}$ is the base of our photosensor device. The gain dispersion between them has been compensated through 4 programmable ASICs, showing the convenience of using that kind of devices as readout electronics for SiPM based PET detectors. In this work, they have also been used to record the planar $X Y$ position.

Two coupling methods between the SiPM array and the monolithic LYSO crystal have been studied. The coupling with 


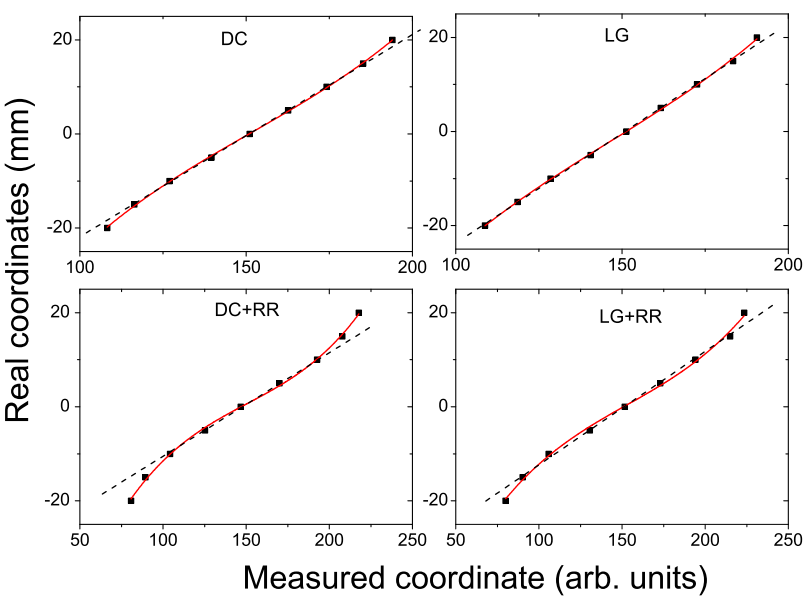

Figure 8: Relationship between measured and real coordinates for the $\mathrm{CoG}^{275}$ the 9 test positions and for the four experimental set-ups. 277

light guides reduces the effect of compression, at the cost $\mathrm{of}_{281}^{280}$ poor statistics that can be partially compensated with the use 282 of a retrorreflector layer at the entrance of the crystal. The di-283 rect coupling approach, nevertheless, shows the best energy and ${ }^{284}$ spatial resolution since light with larger acceptance angle can be ${ }_{286}^{285}$ collected by the system. Errors in estimating the center of grav-287 ity due to the presence of dead area limit the spatial resolution. ${ }^{288}$ Analysis of higher order moments may help to determine the $e^{289}$ degree of symmetry breaking in the sampled distribution, $\operatorname{and}_{291}^{290}$ therefore, improve the spatial resolution. The low scintillation 292 light collection, constrains the energy resolution.

We suggest further studies using smaller SiPMs array pitch, ${ }_{295}^{294}$ thus reducing dead area, improving light collection and there-296 fore both energy and spatial resolutions of the detector block. ${ }^{297}$ Moreover, in the case of coupling through light guides, the $\mathrm{AA}_{299}^{298}$ will also increase.

\section{Acknowledgement}

This work was supported by the Centre for Industrial Techno-305 logical Development co-funded by FEDER through the Tech- ${ }^{307}$ nology Fund (DREAM Project, IDI-20110718), the Spanish $_{309}{ }^{308}$ Plan Nacional de Investigación Científica, Desarrollo e Inno- ${ }_{310}$ vación Tecnológica (I+D+I) under Grant No. FIS2010-21216-311 CO2-01 and the Valencian Local Government under Grant ${ }^{312}$ PROMETEO 2008/114.

\section{References}

[1] S. Moehrs, A.D. Guerra, D.J. Herbert and M.A. Mandelkern, A detector head design for small-animal PET with silicon photomultipliers (SiPM), Phys. Med. Biol. 511113 (2006).

[2] D.R. Schaart, H.T. van Dam, S. Seifert, R. Vinke, P. Dendooeven, H. Löhner and F.J. Beekman, A novel, SiPM-array-based, monolithic sicntillator detector for PET, Phys. Med. Biol. 543501 (2009).
[3] S. España, L.M. Fraile, J.L. Herraiz, J.M. Udías, M. Desco and J.J. Vaquero. Performance evaluation of SiPM photodetectors for PET imaging in the presence of magnetic fields, Nucl. Instrum. Meth. A , 613 308-316 (2010).

[4] M.S. Judenhofer, D.F. Newport, C. Catana, S.B. Siegel, M. Becker, A. Thielscher, M. Kneilling, M.O. Lichy, M. Eichener, K. Klingel, G. Resichl, S. Widmaier, M. Rocken, R.E. Nutt, H.J. Machulla, K. Ulidaq, S.R. Cherry, C.D. Claussen and B.J. Pichler. Simultaneous PETMRI: a new approach for functional and morphological imaging. Nature Medicine, 14 459-465 (2008).

[5] M. R. Ay, S. Sarkar. Computed Tomography Based Attenuation Correction in PET/CT:Principles, Instrumentation, Protocols, Artifacts and Future Trends, Iran J. Nucl. Med. 15(2), 1-29 (2007).

[6] S. Seifert, R. Vinke, H.T. Van Dam, H. Lhner, P. Dendooven, F.J. Beekman and D.R. Schaart. Ultra precise timing with SiPM-Based TOF PET Scintillation Detectors. IEEE 2009 NSS Conference Record (2010).

[7] C. Lerche, J. Benlloch, F. Sánchez, N. Pavón, B. Escat, E. Gimenez, M. Fernández, I. Torres, M. Gimenez, A. Sebastiá and J. Martínez, Depth of gamma-ray interaction within continuous crystals from the width of its scintillation light-distribution, IEEE Trans. Nucl. Sci. 52560 (2005).

[8] J.M. Benlloch, V. Carrilero, A.J. González, J. Catret, C.W. Lerche, D. Abellán, F. García de Quirós, M. Martínez, J. Modia, F. Sánchez, N. Pavón, A. Ros, J. Martínez and A. Sebastiá. Scanner calibration of a small animal PET camera based on continuous LSO crystals and flat panel PSPMTs. Nucl. Instr. and Meth. A 571 26-29 (2007).

[9] A.J. González Martínez, A. Peiró Cloquell, F. Sánchez Martínez, L.F. Vidal San Sebastian and J.M. Benlloch Baviera. Innovative PET detector concept based on SiPMs and continuous crystals, Nucl. Instrum. Meth. A $695213-217$ (2012).

[10] A.J. González, P. Conde, L. Hernández, V. Herrero, J.M. Monzó, A. Orero, A. Peiró, M.J. Rodríguez-Álvarez, A. Ros, F. Sánchez, A. Soriano, L.F. Vidal and J.M. Benlloch. Design of the PETMR system for head imaging of the DREAM Project, Nucl. Instrum. Meth. A 702 94-97 (2013).

[11] J.C. Chaves. Introduction to nonimaging optics, CRC Press, Taylor \& Francis Group, LLC (2008)

[12] V. Herrero-Bosch, C.W. Lerche, M. Spaggiari, R. Aliaga-Varea, N. Ferrando-Jodar and R. Colom-Palero. AMIC: An Expandable Front-End for Gamma-Ray Detectors With Light Distribution Analysis Capabilities, IEEE Trans. Nucl. Sci. 58 1641-1646 (2011).

[13] C.W.Lerche, V. Herrero-Bosch, M. Spaggiari, F. Mateo-Jimenez, J.M. Monzó-Ferrer, R.J. Colom-Palero, F.Mora-Mas. Fast circuit topology for spatial signal distribution analysis. Real Time Conference, 1-8 (2010)

[14] P. Conde, A.J. González, L. Hernández, L. Moliner, A. Orero, M.J. Rodrígue-Álverez, F. Sánchez, A. Soriano, L.F. Vidal and J.M. Benlloch. First results of an ASIC controlled $\gamma$-detector based on a SiPM-array and a monolithic LYSO, IEEE 2012 NSS-MIC Conference Record, 412-414 (2013).

[15] A. Ros, R.J. Aliaga, V. Herrero-Bosch, J.M. Monzo, A. González, R.J. Colom, F.J. Mora and J.M. Benlloch. Expandable Programmable Integrated Front-End for Scintillator Based Photodetectors, IEEE 2012 NSSMIC Conference Record, 3196-3200 (2013).

[16] G. Landi. Properties of the center of gravity as an algorithm for position measurements. Nucl. Instrum. Meth. A 485 698-719 (2002).

[17] C. Lerche, Depth of interatcion enhanced gamma-ray imaging for medial applications, PhD. Thesis, Universidad de Valencia, 2006.

[18] A. Soriano, A.J. González, F. Sánchez, P. Conde, L. Moliner, A. Orero, M.J. Rodríguez-Alvarez, L.F. Vidal and J.M. Benlloch. Minimization of parallax error in breast dedicated PET IEEE Trans. Nucl. Sci. 60 739745 (2013). 